\title{
Laryngeal Papillary Cystadenoma: A Case Report
}

\author{
Min-Kyung Kim ${ }^{1}$, Jiwon Koh² (D), Kyeong Cheon Jung² (D), and Seong Keun Kwon 1,3,4 (D) \\ 'Department of Otorhinolaryngology-Head and Neck Surgery, Seoul National University Hospital, Seoul, Korea \\ 2Department of Pathology, Seoul National University Hospital, Seoul National University College of Medicine, Seoul, Korea \\ ${ }^{3}$ Department of Otorhinolaryngology-Head and Neck Surgery, Seoul National University College of Medicine, Seoul, Korea \\ ${ }^{4}$ Department of Otorhinolaryngology-Head and Neck Surgery, Biomedical Research Institute, Seoul National University Hospital, Seoul, Korea
}

Papillary cystadenoma is a rare, benign salivary gland neoplasm containing cystic cavities with intraluminal papillary projections. In the head and neck area, it occurs mainly in major and intraoral minor salivary glands, but rarely in the larynx. We report a case of a 67-year-old female with a chief complaint of hoarse voice diagnosed as laryngeal papillary cystadenoma. This paper emphasizes the need to consider papillary cystadenoma as one of differential diagnosis when benign looking lesions are observed in the larynx, where it uncommonly occurs.

Keywords Cystadenoma, papillary; Larynx; Laryngeal tumors.

\section{INTRODUCTION}

Cystadenoma is a benign neoplasm which constitutes $0.7 \%$ to $8.1 \%$ of all benign salivary gland tumors. It is composed of multiple cystic spaces lined by variable epithelium with focal intraluminal papillary proliferations. The average age of occurrence is about 50 years with female predominance (female-to-male ratio, 3:1). Most of the cystadenomas arise in major salivary glands, $58 \%$ in parotid gland and $7 \%$ in submandibular gland. Minor salivary gland cystadenomas affect mostly the lip and buccal mucosa [1]. Minor salivary glands tumors are rarely observed in the larynx. They arise from the submucosal glands and constitute less than $1 \%$ of laryngeal tumors [2].

Herein we present a rare case of laryngeal papillary cystadenoma with its histologic features, diagnosis, and treatment process.

\section{CASE REPORT}

A 67-year-old female without remarkable medical history presented with chief complaint of hoarseness which started a month ago. There was no dyspnea, dysphagia nor odynophagia. Laryngeal examination with videostroboscopy revealed a submucosal benign looking granulomatous mass at her left false vocal fold. Both true vocal cords were normal in appearance and mobility. The subglottis was also free (Fig. 1). Routine blood tests were within normal limits. There was no palpable cervical lymphadenopathy. Computed tomography scan revealed approximately 7-mm-sized enhancing nodular lesion at left laryngeal ventricle. There was neither significant cervical lymphadenopathy nor other abnormality in the pharynx and salivary gland (Fig. 2).

To excise and biopsy the supraglottic mass, laryngeal microsurgery was performed un-

\author{
Received June 25, 2021 \\ Revised August 30, 2021 \\ Accepted September 17, 2021 \\ Corresponding Author \\ Seong Keun Kwon, MD, PhD \\ Department of Otorhinolaryngology- \\ Head and Neck Surgery, \\ Seoul National University Hospital, \\ 101 Daehak-ro, Jongno-gu, \\ Seoul 03080, Korea \\ Tel +82-2-2072-2286 \\ Fax+82-2-745-2387 \\ E-mail kwon.seongkeun@gmail.com \\ ORCID iDs \\ Min-Kyung Kim (D) \\ https://orcid.org/0000-0001-9026-682X \\ Jiwon Koh (iD \\ https://orcid.org/0000-0002-7687-6477 \\ Kyeong Cheon Jung (iD) \\ https://orcid.org/0000-0002-7741-7184 \\ Seong Keun Kwon (iD \\ https://orcid.org/0000-0001-9218-7666 \\ This is an Open Access article distributed \\ under the terms of the Creative \\ Commons Attribution Non-Commercial \\ License (https://creativecommons.org/ \\ licenses/by-nc/4.0) which permits \\ unrestricted non-commercial use, \\ distribution, and reproduction in any \\ medium, provided the original work is \\ properly cited.
}


der general anesthesia. Kleinsasser laryngoscope was inserted for suspension exam of larynx. The well-circumscribed soft mass surrounded by granulation tissue was originating from left laryngeal ventricle. The mass was extending to left thyroarytenoid muscle. The supraglottic mass was completely ex-

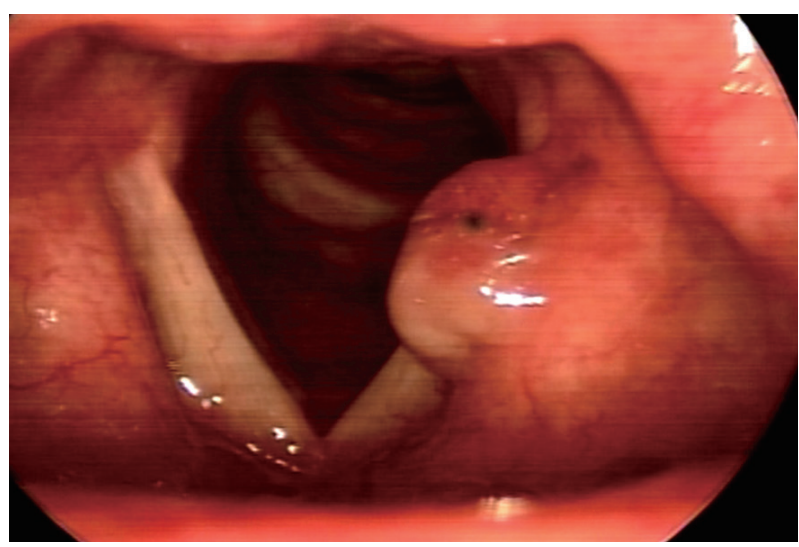

Fig. 1. Videostroboscopic examination of larynx revealed a granulomatous mass in the left false vocal fold.

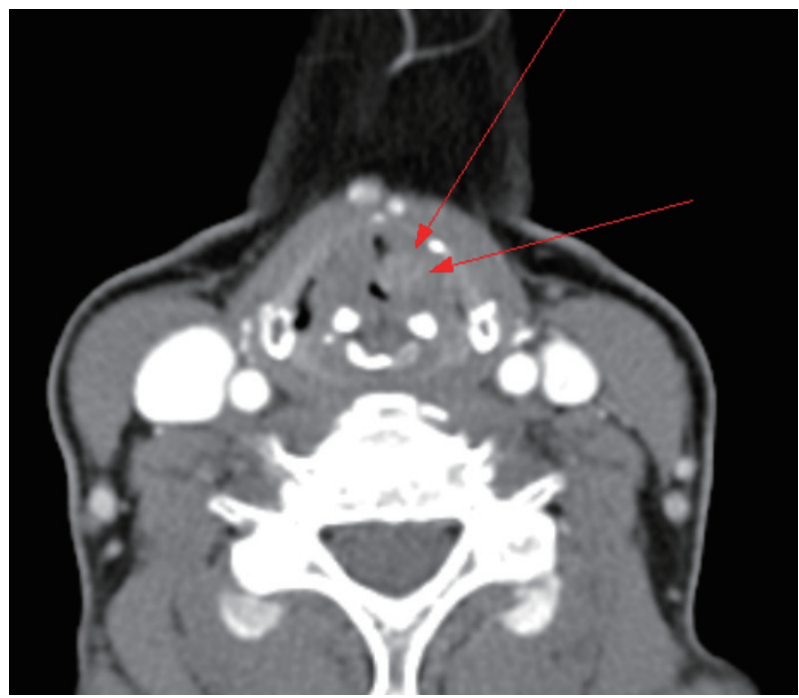

Fig. 2. Contrast-enhanced neck CT revealed approximately 7-mmsized enhancing nodular lesion in the laryngeal ventricle (arrows). cised using $\mathrm{CO}_{2}$ laser, with sufficient safety margin (Fig. 3). A frozen section biopsy identified the mass as a benign lesion. The remaining whole specimen was sent to pathologist for histopathological analysis.

Histopathological analysis revealed submucosal multiseptated cystic lesion measuring $0.7 \mathrm{~cm}$ in the greatest diameter (Fig. $4 \mathrm{~A})$. The cyst was characterized by inner papillary projections, which were lined by columnar epithelial cells (Fig. 4B and C). The cells showed no significant cytologic atypia or mitotic figures (Fig. 4D), and no abnormal invasion into the fibrous capsule was noted. Therefore, the patient was finally diagnosed with papillary cystadenoma (PC).

Postoperative hospital course was uneventful. The patient was followed up for over 1 year with no evidence of recurrence.

\section{DISCUSSION}

As previously described, cystadenomas are rare, benign neoplasms. The rarity of PCs has been reported by many authors. A retrospective study of 6982 cases of salivary gland by Tian et al. [3] reported that cystadenomas account for $0.59 \%$ of benign salivary gland tumors and there was only one case of laryngeal cystadenoma out of 28 cases of cystadenomas. A review of 426 cases of intraoral minor salivary gland tumors by Waldron et al. [4] reported that PC comprises $4.7 \%$ of minor salivary gland tumors. PubMed and Google Scholar database search from 1953 to 2021 revealed only 4 cases of non-oncocytic laryngeal papillary cystadenoma in the English literature [5-8].

Histologically, cystadenomas consist of multicystic proliferation of glandular epithelium which could be oncocytic, apocrine, epidermoid, and mucinous. WHO classification (2005) of salivary gland tumors discriminated two variants of cystadenoma: papillary cystadenoma and mucinous cystadenoma [9]. Mucinous cystadenoma is composed of multiple cysts lined exclusively by mucinous cells. Papillary type cystadenoma as in this case is characterized by cystic lesion with intraluminal papillary projections. The lining epithelial cells may accompany on-
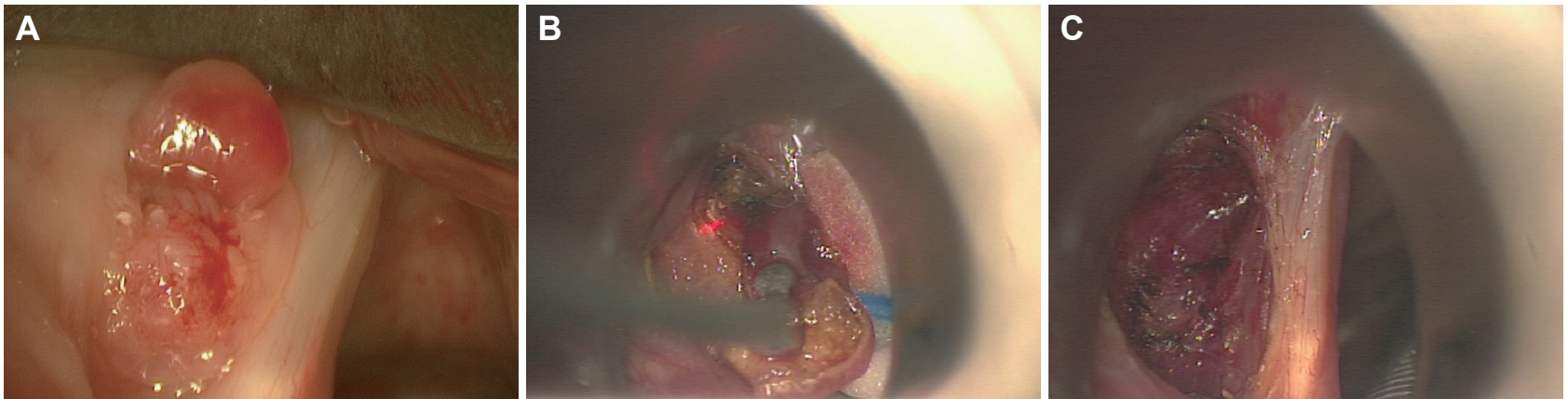

Fig. 3. Operative pictures of the supraglottic mass. A: The mass was extended to the ventricle and true vocal fold. B: Excision of the mass using $\mathrm{CO}_{2}$ laser. C: Postoperative status. 

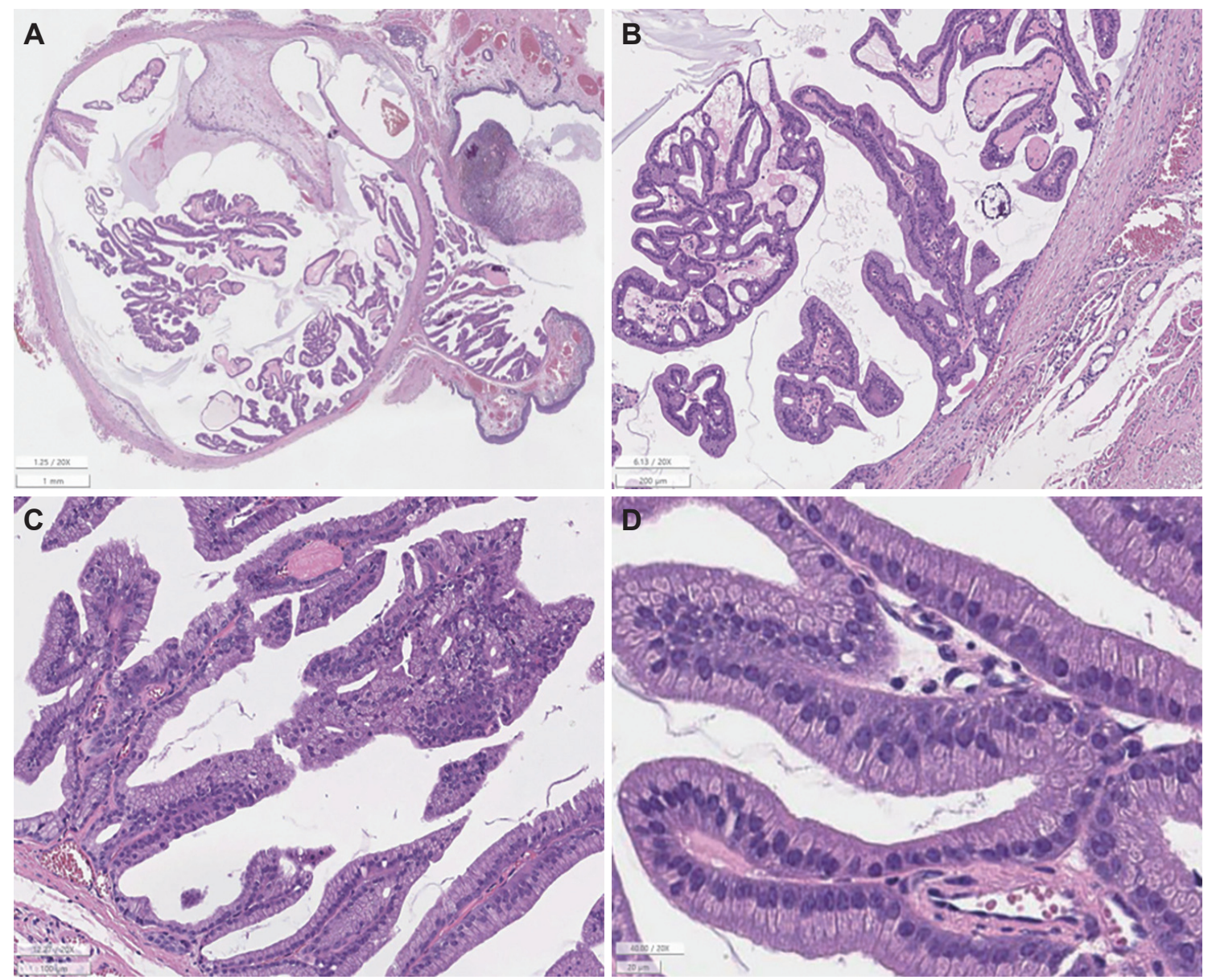

Fig. 4. Histologic features (Hematoxylin-Eosin staining, $\times 20$ ). A: Well-circumscribed multicystic lesion with papillary cores is noted on low power. B, C: Columnar cells with eosinophilic cytoplasm were lining the fibrovascular cores. D: The cells were uniform and bland-looking without any malignant features on magnified view.

cocytic metaplasia which is suspected to be related with age and is facilitated by smoking [1]. However, in this case oncocytic change was not observed.

In the larynx, the most common anatomical site of cystadenoma is the supraglottis (74\%). This is due to abundant distribution of seromucinous salivary glands in laryngeal ventricles and vestibular folds. Hoarseness is the most common symptom. Less frequent symptoms include globus, dysphagia, pain, stridor, and laryngeal obstruction [10].

In our case, laryngeal examination with videostroboscopy revealed the benign looking mass located at supraglottis. Further imaging work up with CT scan and/or MRI may give more information about the extent of the tumor and anatomical relationships. On CT, PCs appear as well defined, homogenous, isodense or slightly hyperdense, and smooth submucosal lesion [11].

The histopathological diagnosis is essential as many cystic lesions of the larynx are with similar property but requires different management. The differential diagnosis of PC involves papillary cystadenoma lymphomatosum (Warthin tumor), oncocytic cystadenomas, oncocytomas, low-grade mucoepidermoid carcinoma, cystadenocarcinoma, and intraductal papilloma.

The treatment of choice for laryngeal PC is complete excision with tumor-free margin as in the treatment of other benign tumors. Given the anatomical site and the size of the tumor, surgical approach could be made transorally or transcervically with endoscopic and/or microscopic assistance. The cases of oncocytic papillary cystadenoma accompanying squamous cell carcinoma have been reported [12]. However, these cases were extremely rare and malignant transformation of oncocytic cystadenoma has not been reported so far [13]. Although PC is a benign lesion, considering its possibility of recurrence and multiple site involvement [14,15], follow-up is recommended. In our case, annual visit was planned starting from 1 year after operation.

We present this rare case of laryngeal PC to emphasize that clinicians should consider this disease as one of the differential diagnosis of laryngeal cystic lesions. In addition, histopathological diagnosis is critical in diagnosing the PC as many salivary gland tumors show similar features. Curative treatment is com- 
plete surgical excision with tumor-free margin.

\section{Acknowledgments}

None

Conflicts of Interest

The authors have no financial conflicts of interest.

\section{Authors' Contribution}

Conceptualization: Seong Keun Kwon. Data curation: Min-Kyung Kim. Formal analysis: Min-Kyung Kim, Jiwon Koh. Investigation: Min-Kyung Kim, Seong Keun Kwon. Methodology: Min-Kyung Kim, Seong Keun Kwon. Project administration: Min-Kyung Kim, Seong Keun Kwon. Resources: Kyeong Cheon Jung, Seong Keun Kwon. Software: Min-Kyung Kim, Seong Keun Kwon. Supervision: Kyeong Cheon Jung, Seong Keun Kwon. Validation: Min-Kyung Kim, Jiwon Koh, Seong Keun Kwon. Visualization: all authors. Writingoriginal draft: Min-Kyung Kim.Writing_review \& editing: Jiwon Koh, Kyeong Cheon Jung, Seong Keun Kwon. Approval of final manuscript: all authors.

\section{REFERENCES}

1. Skalova A. Cystadenoma, Salivary Glands of. In: Volavšek M, editor. Head and Neck Pathology. Cham: Springer International Publishing; 2016. p.95-8.

2. Bak-Pedersen K, Nielsen KO. Subepithelial mucous glands in the adult human larynx. Studies on number, distribution and density. Acta Otolaryngol 1986;102(3-4):341-52.

3. Tian Z, Li L, Wang L, Hu Y, Li J. Salivary gland neoplasms in oral and maxillofacial regions: A 23-year retrospective study of 6982 cases in an eastern Chinese population. Int J Oral Maxillofac Surg 2010;39(3): $235-42$
4. Waldron CA, el-Mofty SK, Gnepp DR. Tumors of the intraoral minor salivary glands: A demographic and histologic study of 426 cases. Oral Surg Oral Med Oral Pathol 1988;66(3):323-33.

5. Rangera D, Thackray AC. Papillary cystadenoma of the larynx. J Laryngol Otol 1953;67(10):609-14.

6. Kuhn AJ. Cystadenoma of the parotid gland and larynx. Arch Otolaryngol 1961;73:404-6.

7. Donald PJ, Krause CJ. Papillary cystadenoma of the larynx. Laryngoscope 1973;83(12):2024-8.

8. Abou Ali T, Al Sabah B, Gopalan K. Papillary cystadenoma of larynx: A case report. International Journal of Otolaryngology and Head \& Neck Surgery 2013;2(6):280-5.

9. Eveson JW, Auclair P, Gnepp DR, El-Naggar AK. Tumours of the salivary glands. In: Barnes L, Eveson JW, Reichart P, Sidransky D. World Health Organization Classification of Tumours. Pathology and Genetics of Head and Neck Tumours. Lyon: IARC Press;2005, p.212-5.

10. Lundgren J, Olofsson J, Hellquist H. Oncocytic lesions of the larynx. Acta Otolaryngol 1982;94(1-6):335-44.

11. Friedman L, Patel M, Steinberg J, Hardy D. CT appearance of an oncocytic papillary cystadenoma of the larynx. J Comput Assist Tomogr 1990;14(2):322-4.

12. Stenner M, Müller KM, Koopmann M, Rudack C. Squamous cell carcinoma of the larynx arising in multifocal pharyngolaryngeal oncocytic papillary cystadenoma: A case report and review of the literature. Medicine (Baltimore) 2014;93(12):e70.

13. Heyes R, Lott DG. Laryngeal cysts in adults: Simplifying classification and management. Otolaryngol Head Neck Surg 2017;157(6):92839.

14. Collins EM. Papillary cystadenoma of accessory salivary gland. Am J Surg 1958;96(6):749-50.

15. LeJeune FE Jr, Putman HC 3rd, Yamase HT. Multiple oncocytic papillary cystadenomas of the larynx: A case report. Laryngoscope 1980; 90(3):501-4. 